\title{
The Factor Structure of the UPDRS as an Index of Disease Progression in Parkinson's Disease
}

\author{
Jonathan R. Evans ${ }^{\mathrm{a}, *}$, Sarah L. Mason ${ }^{\mathrm{a}}$, Caroline H. Williams-Gray ${ }^{\mathrm{a}}$, Thomas Foltynie ${ }^{\mathrm{b}}$, \\ Matthew Trotter ${ }^{\mathrm{c}}$ and Roger A. Barker ${ }^{\mathrm{a}}$ \\ ${ }^{\mathrm{a}}$ Cambridge Centre for Brain Repair, University of Cambridge, Cambridge, UK \\ ${ }^{\mathrm{b}}$ Institute of Neurology, University College London, London, UK \\ ${ }^{\mathrm{c}}$ Anne McLaren Laboratory for Regenerative Medicine, University of Cambridge, Cambridge, UK
}

\begin{abstract}
The optimum method for evaluating disease progression in Parkinson's disease (PD) has not been established, and this has implications for clinical trials. The majority of previous studies have utilized change on the Unified Parkinson's disease Rating Scale (UPDRS) as an index of progression. However, the UPDRS has not been validated for this purpose. We utilized exploratory factor analysis (EFA) to evaluate the longitudinal properties of the UPDRS as an index of disease progression in PD.

Data was derived from a representative cohort of 122 PD patients followed from diagnosis and assessed every 18-24 months for up to 7.9 years. For each subject the rate of change of each item on the UPDRS-3 was calculated and an EFA was performed using this data. Results were compared with those of previously published EFAs in cross-sectional PD cohorts.

The UPDRS-3 retains a stable factor structure when used as an index of disease evolution. The 27 items reduced to 6 factors which accounted for $61.0 \%$ of the variance in disease progression. A dominant factor was identified which incorporated axial (gait/postural stability) symptoms and signs. Our analysis indicates that the UPDRS captures meaningful aspects of disease progression in $\mathrm{PD}$, and that it is possible to identify symptom/sign complexes which evolve independently of one another. Progression in PD is predominantly characterized by the development of axial symptoms and signs. This result has implications for pathogenesis and should also inform natural history models of PD thereby allowing identification of meaningful outcome measures for clinical trials of disease-modifying therapies.
\end{abstract}

Keywords: Parkinson's disease, unified Parkinson's disease Rating Scale (UPDRS), exploratory factor analysis, natural history, disease-modifying

\section{INTRODUCTION}

Our understanding of the natural history of Parkinson's disease (PD) remains incomplete [1]. Although neurodegeneration in PD is insidious and inexorable, the widespread use of dopaminergic replacement therapy ameliorates many of the attendant symptoms and signs and complicates the study of clinical progression.

${ }^{*}$ Correspondence to: Dr. Jonathan R. Evans, Research Registrar in Neurology, Cambridge Centre for Brain Repair, University of Cambridge, Cambridge, UK. E-mail: jonathanevans@doctors. org.uk.
Furthermore, although considerable heterogeneity in the rate of progression between PD sufferers is apparent [2] the reasons for this are unclear. Improving our understanding of the evolution of treated PD is vitally important for a number of reasons:

- To improve prognostication

- To enable titration of PD therapy to maximise clinical benefit at different stages of disease

- To improve the definition of disease staging criteria in PD 
- To allow identification of meaningful outcome measures in clinical trials, particularly of therapies with a putative "disease-modifying" effect.

There is no gold-standard, ante-mortem index of progression in PD. A number of clinical disease rating scales have been developed for use in PD and, although in general designed to evaluate disease burden at a given time-point, it is common practice to use serial measurements upon such scales as either a measure of progression or, conversely, of response to an intervention [3]. The design of clinical rating instruments may of necessity incorporate some arbitrary elements, as disease progression upon a biological continuum is frequently expressed in terms of pre-defined disease stages. Deficiencies in the sensitivity, specificity, reliability or validity of such scales may reduce their effectiveness as indices of disease progression.

The most widely used assessment scale in PD is the Unified Parkinson's Disease Rating Scale (UPDRS) [4]. Psychometric evaluation of the UPDRS has shown it to be reliable and internally consistent $[5,6]$. The statistical technique of factor analysis has previously been used to investigate the metric properties of the motor section of the UPDRS (UPDRS-3) which has been shown to have a discreet factor structure [7-9], i.e. groups of symptoms/signs appear to cluster into separate domains. However, these studies have been conducted in cross-sectional PD cohorts featuring patients with varying disease durations. No previous studies have sought to study the metric properties of the UPDRS when used as a tool to measure progression in PD over time, but such an analysis would be valuable for two reasons. First, given the widespread use of the UPDRS in longitudinal and interventional studies, it is important that its validity in this indication is established objectively. Secondly, it would allow a more general description of the pattern of evolution of symptoms and signs in PD, thereby enhancing our knowledge of the natural history of PD.

In this study we have addressed this issue in a population-representative PD cohort followed longitudinally from diagnosis. Exploratory factor analysis (EFA) has been applied not to absolute UPDRS-3 scores, but to the rate of change in each UPDRS-3 item over time with the aim of evaluating whether particular clusters of symptoms/signs progress at different rates. This data-driven, hypothesis-free approach allows an objective evaluation of the pattern of development of symptom-sign complexes in treated PD.

\section{METHODS}

\section{Subjects}

Data for this analysis was derived from participants in a community-based longitudinal study of incident PD in the county of Cambridgeshire, UK. The study met with the approval of the local ethics committee and all patients provided written, informed consent. The methods used for case ascertainment have been described previously [10].

Subjects have been followed from the point of diagnosis at 18-24 month intervals using a comprehensive clinical battery including the UPDRS [11]. Items on the UPDRS were identified using the convention adopted by Stebbins et al. [7], so that the motor section (UPDRS section 3) corresponded to items 17-44 inclusive. UPDRS Assessments were performed in the subjective "On" state and were conducted either at our research facility, or as home visits so as to minimise drop-out. UKPDS Brain Bank criteria [12] were used to diagnose PD, and these were re-applied at followup round 2 to minimise clinical mis-diagnosis. Subjects were followed until death or withdrawal. Only subjects assessed on 3 or more occasions have been included in this analysis to minimise bias. Management and treatment decisions for each participant continued to be at the discretion of their supervising physician and were not under the control of the study investigators.

\section{Statistical analysis}

For each subject, the rate of change in each item of the UPDRS section 3 was calculated as:

\section{(Final score-Initial Score)/Total follow-up period (years)}

The follow-up period was expressed as the exact interval between initial and final assessments, without rounding. This produced a matrix of 27 variables for each subject which were then entered into the EFA. Histogram plots of the distribution of these variables were inspected to ensure that the assumption of Normality was satisfied.

Factor analysis was performed using a principal components method over a maximum of 25 iterations, and an oblique rotation using the direct oblimin algorithm was used to aid factor discrimination. Kaiser's criteria of returning all factors with eigenvalues $>1$ was used in the initial analysis [13]. This was then refined by inspection of a scree plot of the eigenvalues of factors added consecutively to the solution as described 
by Field [14]. The analysis was then re-run with the optimal factor structure specified, and the resultant pattern matrix evaluated with factor loadings of $<0.4$ suppressed to facilitate interpretation.

Sampling adequacy was assessed with the KaiserMeyer-Olkin (KMO) statistic, and the data-set was screened for multicolinearity by inspection of the R-matrix. Bartlett's test of sphericity was used to test the null hypothesis that the R-matrix was an identity matrix. Consistency of the factor solution was assessed using Cronbach's alpha statistic.

All statistical analysis was performed in SPSS v 14.0 (SPSS Inc. Chicago, Ill.).

\section{RESULTS}

The process of case identification from the incident cohort is summarised in Fig. 1. Of 159 patients recruited, 126 patients were available for assessment at follow-up round 2 and still met diagnostic criteria for PD. Four patients were subsequently excluded, meaning that follow-up data from 122 patients from the original cohort was evaluated. Mean length of followup was 5.5 years (1.4), equating to a mean of $4.3(0.8)$ assessments. The clinical characteristics of participants at baseline and at final assessment are displayed in Table 1.

\section{Characteristics of the factor solution}

The KMO statistic was 0.747 , indicating that sample size for this analysis was adequate [15]. There was no evidence of significant multi-colinearity within the dataset (determinant of the R-matrix $=9.2 \mathrm{E}-04$ ). Furthermore, inspection of the individual between item Pearson correlation coefficients showed no values greater than 0.7 .

Bartlett's test was significant at the $p<0.0001$ level, indicating no significant sphericity.

\section{Factor structure}

Eight factors were identified with eigenvalues greater than one. Examination of the scree plot showed an inflection point after the sixth factor which was therefore chosen as the optimal solution. This solution accounted for $61 \%$ of the total variance in the dataset. The factor loadings for each UPDRS item are shown in Table 2.

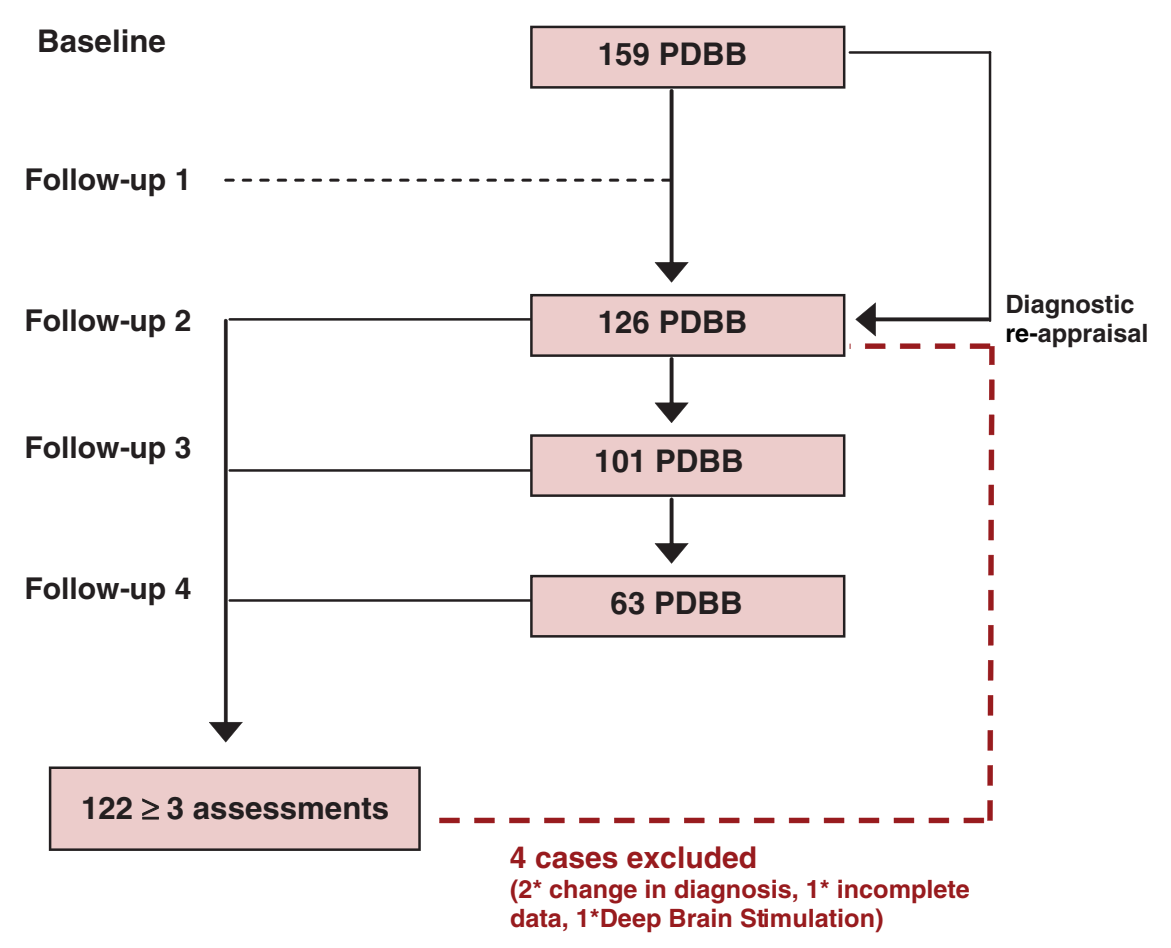

Dec 2000-

Dec 2002

Aug 2001-

Aug 2002

Dec 2004-

Dec 2005

Oct 2006-

April 2007

April 2008-

Feb 2009

TIMELINE

Fig. 1. Flow-chart illustrating the process of case identification for the present study. one hundred and fifty nine patients fulfilled brain bank criteria for PD at entry (PDBB). Of those available for assessment at follow-up round 2, 126 still met brain bank criteria and were eligible for inclusion in the present analysis, although 4 patients were subsequently excluded for the reasons indicated. 
Table 1

Clinical characteristics of the study population

\begin{tabular}{lcc}
\hline & Baseline & Final assessment \\
\hline $\mathrm{M}: \mathrm{F}$ & $70: 52$ & - \\
Age at diagnosis & $69.5(9.8)$ & - \\
Follow-up (years) & $5.5(1.4)$ & - \\
Number of FU assessments & $4.3(0.8)$ & - \\
UPDRS (Total) & $38.4(17.0)$ & $62.3(23.8)$ \\
UPDRS (Motor/Section 3) & $25.9(12.3)$ & $38.2(14.7)$ \\
Hoehn-Yahr Stage & $2(1-3)$ & $2(2-4)$ \\
Medication dose (LED, mg) & $207.0(158.1)$ & $560.1(413.7)$ \\
MMSE & $29.1(0.7)$ & $24.9(6.0)$ \\
\hline
\end{tabular}

Data is in the form mean (sd), except for ${ }^{\dagger}$ median (range). LED, Levodopa equivalent dose, calculated using an algorithm adapted from Brodsky et al. [43] as previously described [11]. MMSE, MiniMental State Examination.
The factor matrix has a defined internal structure with a clustering of UPDRS-3 items which is readily interpretable on clinical grounds:

- Factor 1: Axial items (gait, posture, balance), speech and hypomimia (25.5\% of total variance)

- Factor 2: Items assessing upper limb bradykinesia $(10.0 \%)$

- Factor 3: Items assessing lower limb bradykinesia, but with some overlap with axial items (neck rigidity, central bradykinesia, posture) $(8.5 \%)$

- Factor 4: Items assessing action/postural tremor and lower limb resting tremor $(7.1 \%)$

Table 2

Factor solution for the UPDRS-3 as an index of disease progression. Factor loadings are shown for the optimal, 6-factor solution using principal components analysis with direct oblimin rotation. The output of the pattern matrix is shown. Factor loadings $<0.4$ have been suppressed

\begin{tabular}{|c|c|c|c|c|c|c|}
\hline \multirow[b]{3}{*}{$\%$ variance } & \multicolumn{6}{|c|}{ Components } \\
\hline & 1 & 2 & 3 & 4 & 5 & 6 \\
\hline & $25.5 \%$ & $10.0 \%$ & $8.5 \%$ & $7.1 \%$ & $5.0 \%$ & $4.6 \%$ \\
\hline Cronbach's alpha & 0.835 & 0.797 & 0.769 & 0.733 & 0.725 & 0.657 \\
\hline 18-Speech & 0.554 & & & & & \\
\hline 19-Hypomimia & 0.455 & & & & & \\
\hline \multicolumn{7}{|l|}{ Rest Tremor } \\
\hline 20-Facial & & & & & 0.640 & \\
\hline 21-Right UE & & & & & 0.572 & \\
\hline 22-Left UE & & & & & 0.622 & \\
\hline 23-Right LE & & & & 0.629 & & \\
\hline 24-Left LE & & & & 0.575 & & \\
\hline \multicolumn{7}{|l|}{ Action/Postural Tremor } \\
\hline 25-Right & & & & 0.687 & & \\
\hline 26-Left & & & & 0.698 & & \\
\hline \multicolumn{7}{|l|}{ Rigidity } \\
\hline 27-Neck & & & 0.446 & & & \\
\hline 28-Right UE & & & & & & 0.576 \\
\hline 29-Left UE & & & & & & 0.729 \\
\hline 30-Right LE & & & & & & 0.424 \\
\hline 31-Left LE & & & & & & 0.618 \\
\hline \multicolumn{7}{|l|}{ Finger taps } \\
\hline 32-Right & & 0.844 & & & & \\
\hline 33-Left & & & & & 0.675 & \\
\hline \multicolumn{7}{|l|}{ Hand flashes } \\
\hline 34-Right & & 0.860 & & & & \\
\hline 35-Left & & 0.566 & & & & \\
\hline \multicolumn{7}{|l|}{ Pro-/Supination } \\
\hline 36-Right & & 0.745 & & & & \\
\hline 37-Left & & 0.608 & & & & \\
\hline \multicolumn{7}{|l|}{ Foot taps } \\
\hline 38-Right & & & 0.526 & & & \\
\hline 39-Left & & & 0.730 & & & \\
\hline 40-Arise from chair & 0.756 & & & & & \\
\hline 41-Posture & 0.687 & & 0.402 & & & \\
\hline 42-Gait & 0.774 & & & & & \\
\hline 43-Postural Stability & 0.813 & & & & & \\
\hline 44-Body bradykinesia & 0.606 & & 0.414 & & & \\
\hline
\end{tabular}


- Factor 5: Items assessing upper limb resting tremor $(5.0 \%)$

- Factor 6: Items assessing limb rigidity (4.6\%).

Analysis of between factor correlations is shown in Table 3. Only a small-moderate association between factors was observed (absolute range 0.015-0.251) indicating that they are each capturing separate aspects of disease progression.

\section{Reliability analysis}

Overall internal consistency was high, with Cronbach's alpha $=0.876$, although our analysis clearly suggested that the dataset was multidimensional. As shown in Table 2, individual factors extracted in the analysis also showed a high degree of consistency, with a value of $>0.8$ for the most discriminating factor, factor 1 , and $>0.7$ for the others, excepting factor 6 (0.657). However, this factor explains only $4.6 \%$ of the total variance and, overall, these results would meet recommendations for a reliable solution [16].

\section{DISCUSSION}

Factor analysis of change in the motor section of the UPDRS as a longitudinal measure of progression in PD generated a clinically meaningful solution, with the 27 scale items reducing to 6 factors. Correlation between the identified factors was modest, which suggests that progression in PD may be dissociable into separate domains that we might term "vectors of change". Factor 1, measuring progression in axial and gait dysfunction, accounted for most variance in the dataset. This is consistent with previous reports that postural and gait symptoms, which are relatively refractory to standard dopaminergic therapies [17], tend to progress inexorably with time in PD [18]. Evolution in these domains may be the most meaningful index of progression in treated PD. Aspects of PD progression in the axial domain are captured by the five-item Hoehn

Table 3

Correlation matrix for the rotated factor solution depicted in Table 1. Pearson correlation coefficients for pair-wise comparisons between each component identified in the 6 factor solution are shown

\begin{tabular}{lcccccc}
\hline Component & 1 & 2 & 3 & 4 & 5 & 6 \\
\hline 1 & - & 0.251 & 0.202 & 0.132 & -0.015 & 0.242 \\
2 & 0.251 & - & 0.061 & 0.171 & 0.060 & 0.158 \\
3 & 0.202 & 0.061 & - & 0.114 & 0.090 & 0.015 \\
4 & 0.132 & 0.171 & 0.114 & - & 0.197 & 0.212 \\
5 & -0.015 & 0.060 & 0.090 & 0.197 & - & 0.057 \\
6 & 0.242 & 0.158 & 0.015 & 0.212 & 0.057 & - \\
\hline
\end{tabular}

and Yahr scale (HYS) [19]. Although the HYS is insensitive as an index of disease progression, our analysis would support the view that entry to HYS stage 3 (onset of postural instability) is of prognostic importance in PD [20, 21].

Comparing the present result with that of the two previous studies which have applied EFA to crosssectional cohorts using the entire UPDRS-3 is of obvious interest. In separate studies of 200 patients in the "On", and 294 patients in the "Off" state, Stebbins et al identified very similar factor structures in both conditions [7, 8]. As Table 4 illustrates, the factor solution from our longitudinal analysis matches up with the factor structure determined by cross-sectional analysis in several key areas. This indicates that the UPDRS retains a stable factor structure over time, demonstrating so-called "longitudinal construct validity" [22], which therefore validates its use as a clinical measure of longitudinal progression in PD. This result complements a recent comparative study of rating scales in PD which reported that UPDRS-3 was a sensitive index of progression [23]. Although the UPDRS has recently been superseded by the revised MDS-UPDRS [24], it is still used widely in ongoing clinical trials and naturalistic studies. Furthermore, the basic form of section 3 in the revised version is very similar and it would not be unreasonable to extrapolate this result to the MDS-UPDRS.

The clinical phenotype in PD is heterogeneous [25], but the majority of previous descriptions of this heterogeneity have focused on assessments made at a single

Table 4

Comparison of factor structure of the UPDRS in cross-sectional and longitudinal analyses. Cross-sectional data is derived from two papers published by Stebbins et al exploring the UPDRS structure in the "On" [7] and "Off" [8] state. Longitudinal data is derived from the present analysis. Percentage values indicate the proportion of the total variance in UPDRS scores between subjects explained by each extracted factor. Higher proportions indicate symptom/sign complexes which differ most between subjects either in terms of their absolute value (cross-sectional) or their rate of change with time (longitudinal). Inferentially, it is factors with high values that account principally for phenotypic heterogeneity in PD

\begin{tabular}{|c|c|c|c|c|}
\hline \multirow[t]{2}{*}{ Factor } & & \multicolumn{2}{|c|}{ Cross-sectional } & \multirow{2}{*}{$\begin{array}{l}\text { Longitudinal } \\
\text { (present study }\end{array}$} \\
\hline & & "On" (\%) & "Off" (\%) & \\
\hline 1: Axial/Speech & & 44 & 31 & $25.5 \%$ \\
\hline 2: Resting tremor & & 14 & 14 & $5.0 \%$ \\
\hline 3: Rigidity & & 6 & 7 & $4.6 \%$ \\
\hline \multirow[t]{2}{*}{ 4/5: Bradykinesia } & Left & 5 & 5 & $10.0 \%(\mathrm{UL})^{*}$ \\
\hline & Right & 5 & 8 & $8.5(\mathrm{LL})^{*}$ \\
\hline 6: Action tremor & & 4 & 5 & $7.1 \%^{\dagger}$ \\
\hline
\end{tabular}

UL, Upper limb; LL, Lower limb. ${ }^{*}$ In the longitudinal analysis bradykinesia is separable into UL and LL components rather than $\mathrm{L} / \mathrm{R}$ components. ${ }^{\dagger}$ Also incorporating LL rest tremor 
time point and have not looked specifically at differences in the rate or pattern of progression over time [26-29]. By comparing the longitudinal and crosssectional factor structure of the UPDRS we can gain several insights into the nature of PD heterogeneity. For example, in both Stebbins' and our analyses the same seven UPDRS items load on to a putative axial/speech factor which, in each case, accounts for the most variance. This suggests that between patient variation in the degree of axial symptom burden is the most important contributor to PD heterogeneity at a given point in time, and also that variation in the rate of progression of these axial symptoms is the major contributor to heterogeneity in the longitudinal evolution of PD. In contrast, in our longitudinal analysis resting tremor terms account for far less variance than the corresponding factor in the cross-sectional analysis (5\% vs $14 \%$ ). This indicates that, studied over time, tremor scores either remain largely static or change at similar rates in all subjects, for which there is evidence from other naturalistic studies [30]. Tremor contributes comparatively little to the heterogeneity of PD when studied longitudinally and although it has previously been suggested that patients with a so-called "tremor-dominant" phenotype have a relatively benign prognosis [31], this would not be supported by the present analysis.

In the cross sectional analysis right and left-sided bradykinesia terms are dissociable, whereas in the longitudinal analysis progression in bradykinesia separates instead into upper and lower limb components, with the latter having some overlap with axial items. The suggestion that upper and lower body bradykinesia may progress differentially is an interesting and unanticipated finding. By contrast, whilst some investigators have suggested that symptom laterality in PD may make important contribution to disease heterogeneity [32], this current analysis would suggest laterality is of no prognostic importance.

One interpretation of the present analysis would be that clinical progression in PD can be separated into discreet domains, or vectors. One could propose the existence of at least two such vectors, one capturing axial symptoms/signs and another bradykinesia (rigidity terms, whilst separable, do not account for significant variance in either longitudinal or crosssectional factor structures). There is evidence from both imaging and clinicopathological studies to support the neuroanatomical separation of PD symptom/sign complexes. Bradykinesia has been shown to correlate best with nigrostriatal dopamine deficiency [33] whereas axial and gait dysfunction may result from disease involvement of caudal brainstem loci, such as the pedunculopontine nucleus [34]. Furthermore, patients with prominent tremor have been shown to have increased metabolic activity in the pons and thalamus [35]. Although any such separation is likely to be relative rather than absolute, as we still do not know what drives neurodegeneration in PD it is possible that certain factors may have a selective influence upon progression in particular PD symptom domains. For example, increased age at onset has been associated with the more rapid evolution of axial and gait symptoms [36], and this has implications for how we may best utilise disease-modifying therapies in the future.

The strengths of this study include the use of a community-based cohort which is more likely to be representative of the general PD population. The majority of previous natural history studies of PD have been performed in secondary or tertiary care settings where selection bias may occur [21, 37, 38]. Given the size of the cohort, the length of follow-up is also a strength for a study of this nature, and through the use of home assessments where required, we have endeavoured to minimize bias due to selective case attrition. Finally, by following patients from diagnosis this study provides data of clear prognostic utility, by providing an objective analysis of how the disease evolves from the point of onset.

We opted to study patients in the subjective "On" state for a number of reasons. First, assessing patients in their optimum clinical state means that the resulting outcome data represents the best that can be achieved with conventional treatment, which should be the baseline against which to measure the efficacy of putative disease-modifying therapies. Secondly, evidence from the ELLDOPA Study indicates that levodopa itself may itself have a disease-modifying effect, or at least a prolonged symptomatic effect [39]. Assessing medicated patients in the "Off" state is not equivalent to assessing never-medicated patients. Finally, this made the study more acceptable to participants, as indicated by the relatively low case attrition rate over a length follow-up period. Whilst a criticism that might reasonably be made is that our results may reflect variations in treatment response in addition to differences in disease progression per se, we would argue that this approach is less likely to introduce bias.

Further criticisms of the present study are that the sample size was small relative to the number of variables entered into the factor analysis, although criteria for sampling adequacy were achieved. The rate of change on each UPDRS item was calculated as a linear gradient between initial and final assessments, whereas both imaging and pathological studies have suggested 
that progression may slow with time $[40,41]$. However, a single study which modelled PD progression on clinimetric scales reported the rate of change was not significantly different from linearity [42] and this decision is unlikely to have had a major bearing upon our result. Whilst the use of gradients of change is superior to the use of raw item level data, as the numerator "change in item score" remains ordinal, the variables entered in to the EFA were not truly continuous and this may have had a small influence upon our result.

As a concluding comment, the results presented here should be considered in the context of an exploratory factor analysis. Whilst the raw results of EFA are datadriven and objective, interpretation of these results is subjective, and therefore subject to bias, and the findings presented here require validation in future studies in novel cohorts. Furthermore, our analysis was restricted to those aspects of the disease captured by the UPDRS section 3 and did not evaluate progression in non-motor domains which would also be amenable to study using the methods described. We have presented preliminary evidence to suggest that progression in $\mathrm{PD}$ may be dissociable into different components. Differences in the rate of progression in relatively treatmentresistant axial symptoms and signs account for most of the heterogeneity between sufferers when studied longitudinally. This has clear implications for pathophysiological modelling of the natural history of PD and, furthermore, should inform the design of outcome measures in forthcoming clinical trials as we enter the era of potentially disease-modifying therapies.

\section{ACKNOWLEDGMENTS}

This work was made possible by the award of an NIHR award to Addenbrooke's Hospital and the University of Cambridge as well as support from CURE-PD and Parkinson's UK. J.R Evans is supported by the Van Geest Foundation through a Gussy Marlowe Clinician Fellowship, and is in receipt of a Raymond and Beverley Sackler Studentship.

\section{REFERENCES}

[1] Poewe W (2006) The natural history of Parkinson's disease. J Neurol, 253 (Suppl 7), VII2-VII6.

[2] Maetzler W, Liepelt I, \& Berg D (2009) Progression of Parkinson's disease in the clinical phase: potential markers. Lancet Neurol, 8, 1158-1171.

[3] Ramaker C, Marinus J, Stiggelbout AM, \& Van Hilten BJ (2002) Systematic evaluation of rating scales for impairment and disability in Parkinson's disease. Mov Disord, 17, 867 876.
[4] Fahn S (1987) ERL: Unified parkinson's disease rating scale; In Recent developments in parkinson's Disease, Fahn S. ERL, Marsend CD, Goldstein M, Calne DB. eds, Macmillan Healthcare Information, vol 2, pp. 153-163.

[5] van Hilten JJ, van der Zwan AD, Zwinderman AH, \& Roos RA (1994) Rating impairment and disability in Parkinson's disease: evaluation of the unified Parkinson's disease rating scale. Mov Disord, 9, 84-88.

[6] Martinez-Martin P, Gil-Nagel A, Gracia LM, Gomez JB, Martinez-Sarries J, \& Bermejo F (1994) Unified Parkinson's disease rating scale characteristics and structure. The cooperative multicentric group, Mov Disord, 9, 76-83.

[7] Stebbins GT, \& Goetz CG (1998) Factor structure of the unified Parkinson's disease rating scale: motor examination section. Mov Disord, 13, 633-636.

[8] Stebbins GT, Goetz CG, Lang AE, \& Cubo E (1999) Factor analysis of the motor section of the unified Parkinson's disease rating scale during the off-state. Mov Disord, 14, 585-589.

[9] Louis ED, Tang MX, \& Mayeux R (2004) Factor structure of Parkinsonian signs in the community-dwelling elderly. Mov Disord, 19, 268-272.

[10] Foltynie T, Brayne CE, Robbins TW, \& Barker RA (2004) The cognitive ability of an incident cohort of Parkinson's patients in the UK. The Campaign Study. Brain, 127, 550-560.

[11] Williams-Gray CH, Foltynie T, Brayne CE, Robbins TW, \& Barker RA (2007) Evolution of cognitive dysfunction in an incident Parkinson's disease cohort. Brain, 130, 1787-1798.

[12] Gibb WR \& Lees AJ (1988) The relevance of the lewy body to the pathogenesis of idiopathic Parkinson's disease. J Neurol Neurosurg Psychiatry, 51, 745-752.

[13] Kaiser HF (1960) The application of electronic computers to factor analysis. Educational and Psychological Measurement, 41, 133-145.

[14] Field A (2005) Exploratory factor analysis; Discovering statistics using spss, SAGE Publications Ltd, pp. 619-679.

[15] Hutcheson GaS N (1999) The multivariate social scientist. SAGE publications Ltd., London, UK, pp. 224-225.

[16] Cortina JM (1993) What is coefficient aplha? An examination of theory and applications. Journal of Applied Pscyhology, 78, 98-104.

[17] Sethi K (2008) Levodopa unresponsive symptoms in Parkinson disease. Mov Disord, 23 (Suppl 3), S521-S533.

[18] Hely MA, Morris JG, Reid WG, \& Trafficante R (2005) Sydney multicenter study of Parkinson's disease: non-1-doparesponsive problems dominate at 15 years. Mov Disord, 20, 190-199.

[19] Hoehn MM, \& Yahr MD (1967) Parkinsonism: onset, progression and mortality. Neurology, 17, 427-442.

[20] Goetz CG, Poewe W, Rascol O, Sampaio C, Stebbins GT, Counsell C, Giladi N, Holloway RG, Moore CG, Wenning GK, Yahr MD, \& Seidl L (2004) Movement disorder society task force report on the hoehn and yahr staging scale: Status and recommendations. Mov Disord, 19, 1020-1028.

[21] Goetz CG, Stebbins GT, \& Blasucci LM (2000) Differential progression of motor impairment in levodopa-treated Parkinson's disease. Mov Disord, 15, 479-484.

[22] Terwee CB, Dekker FW, Wiersinga WM, Prummel MF, \& Bossuyt PM (2003) On assessing responsiveness of healthrelated quality of life instruments: guidelines for instrument evaluation. Qual Life Res, 12, 349-362.

[23] Schrag A, Spottke A, Quinn NP, \& Dodel R (2009) Comparative responsiveness of Parkinson's disease scales to change over time. Mov Disord, 24, 813-818.

[24] Goetz CG, Fahn S, Martinez-Martin P, Poewe W, Sampaio C, Stebbins GT, Stern MB, Tilley BC, Dodel R, Dubois B, 
Holloway R, Jankovic J, Kulisevsky J, Lang AE, Lees A, Leurgans S, LeWitt PA, Nyenhuis D, Olanow CW, Rascol O, Schrag A, Teresi JA, Van Hilten JJ, \& LaPelle N (2007) Movement disorder society-sponsored revision of the unified Parkinson's disease rating scale (MDS-UPDRS): process, format, and clinimetric testing plan. Mov Disord, 22, 41-47.

[25] Foltynie T, Brayne C, \& Barker RA (2002) The heterogeneity of idiopathic Parkinson's disease. J Neurol, 249, 138-145.

[26] Lewis SJ, Foltynie T, Blackwell AD, Robbins TW, Owen AM, \& Barker RA (2005) Heterogeneity of Parkinson's disease in the early clinical stages using a data driven approach. J Neurol Neurosurg Psychiatry, 76, 343-348.

[27] Zetusky WJ, Jankovic J, \& Pirozzolo FJ (1985) The heterogeneity of Parkinson's disease: clinical and prognostic implications. Neurology, 35, 522-526.

[28] Graham JM \& Sagar HJ (1999) A data-driven approach to the study of heterogeneity in idiopathic Parkinson's disease: Identification of three distinct subtypes. Mov Disord, 14, 10-20.

[29] Bostantjopoulou S, Logothetis J, Katsarou Z, \& Mentenopoulos G (1991) Clinical observations in early and late onset Parkinson's disease. Funct Neurol, 6, 145-149.

[30] Louis ED, Tang MX, Cote L, Alfaro B, Mejia H, \& Marde K (1999) Progression of Parkinsonian signs in Parkinson disease. Arch Neurol, 56, 334-337.

[31] Jankovic J, Rajput AH, McDermott MP, \& Perl DP (2000) The evolution of diagnosis in early Parkinson disease. Parkinson Study Group. Arch Neurol, 57, 369-372.

[32] Djaldetti R, Ziv I, \& Melamed E (2006) The mystery of motor asymmetry in Parkinson's disease. Lancet Neurol, 5, 796-802.

[33] Vingerhoets FJ, Schulzer M, Calne DB, \& Snow BJ (1997) Which clinical sign of Parkinson's disease best reflects the nigrostriatal lesion? Ann Neurol, 41, 58-64.
[34] Pahapill PA \& Lozano AM (2000) The pedunculopontine nucleus and Parkinson's disease. Brain, 123(Pt 9), 1767 1783.

[35] Antonini A, Moeller JR, Nakamura T, Spetsieris P, Dhawan V, \& Eidelberg D (1998) The metabolic anatomy of tremor in Parkinson's disease. Neurology, 51, 803-810.

[36] Levy G (2007) The relationship of Parkinson disease with aging. Arch Neurol, 64, 1242-1246.

[37] Schrag A, Dodel R, Spottke A, Bornschein B, Siebert U, \& Quinn NP (2007) Rate of clinical progression in Parkinson's disease. A prospective study. Mov Disord, 22, 938-945.

[38] Jankovic J, \& Kapadia AS (2001) Functional decline in Parkinson disease. Arch Neurol, 58, 1611-1615.

[39] Fahn S, Oakes D, Shoulson I, Kieburtz K, Rudolph A, Lang A, Olanow CW, Tanner C, \& Marek K (2004) Levodopa and the progression of Parkinson's disease. $N$ Engl J Med, 351 , 2498-2508.

[40] Kish SJ, Shannak K, Rajput A, Deck JH, \& Hornykiewicz O (1992) Aging produces a specific pattern of striatal dopamine loss: implications for the etiology of idiopathic Parkinson's disease. J Neurochem, 58, 642-648.

[41] Pirker W, Holler I, Gerschlager W, Asenbaum S, Zettinig G, \& Brucke T (2003) Measuring the rate of progression of Parkinson's disease over a 5 -year period with beta-cit spect. Mov Disord, 18, 1266-1272.

[42] Schulzer M, Lee CS, Mak EK, Vingerhoets FJ, \& Calne DB (1994) A mathematical model of pathogenesis in idiopathic parkinsonism. Brain, 117(Pt 3), 509-516.

[43] Brodsky MA, Godbold J, Roth T, \& Olanow CW (2003) Sleepiness in Parkinson's disease: A Controlled Study. Mov Disord, 18, 668-672. 